\title{
In Vitro Susceptibility Testing of Aspergillus and Non-Aspergillus Filamentous Moulds to Antifungal Agents: Evaluation of Three Different Methods
}

\author{
Gordana Mirchevska ${ }^{1}$, Philipp P. Bosshard ${ }^{2}$ \\ IInstitute of Microbiology and Parasitology, Faculty of Medicine, University "Ss Cyril and Methodius", Skopje, Republic of Macedonia; \\ ${ }^{2}$ Department of Dermatology, University Hospital Zurich, Zurich, Switzerland
}

\begin{abstract}
Citation: Mirchevska G, Bosshard PP. In Vitro Susceptibility Testing of Aspergillus and NonAspergillus Filamentous Moulds to Antifungal Agents: Evaluation of Three Different Methods. Maced J Med Sci. 2012 Oct 15: 5(3):280-287. Maced J Med Sci. 2012 Oct 15, 5(3):280-287. http://dx.doi.org/10.3889/MJMS.1857 5773.2012.0257.

Key words: Aspergillus; filamentous fungi; antifungals; resistance; susceptibility testing.

Correspondence: Dr. Gordana Mirchevska. Institute of Microbiology and Parasitology, Faculty of Medicine, University "Ss Cyril and Methodius" of Macedonia. E-mail: gordmir@yahoo.com

Received: 03-Jul-2011; Revised: 19-Sep-2011; Accepted: 20-Oct-2011; Online first: 23-Sep-2012

Copyright: () 2012 Mirchevska G. This is an open-access article distributed under the terms of the Creative Commons Attribution License, which permits unrestricted use, distribution, ar reproductionin anymedium, provide

Competing Interests: The author have declared that no competing interests exist.
\end{abstract}

\section{Abstract}

Aim: The aim of this study was to evaluate the suitability of Sensititre YeastOne (SYO) method for susceptibility profiles' determination of non-Aspergillus moulds and of E-test for Aspergillus spp. and non-Aspergillus moulds, in comparison with the M38-A reference broth microdilution (BMD) method.

Material and Methods: A total of 33 clinical isolates of filamentous fungi were tested.

Results: The agreement between E-test and BMD at \pm 2 dilutions was $82.4 \%, 83.3 \%$ and $82.4 \%$ for amphotericin B, itraconazole and voriconazole, respectively. The agreement between SYO and BMD at \pm 2 dilutions was $76.5 \%, 66.7 \%$ and $88.2 \%$ for amphotericin $\mathrm{B}$, itraconazole and voriconazole, respectively. The majority of differing results are due to higher MICs with the reference method. In particular, SYO was unable to detect some of the potentially amphotericin B resistant strains. We found that both E-test and SYO method were reproducible and served as suitable methods for antifungal susceptibility testing of moulds.

Conclusion: In conclusion, both E-test and SYO method are promising, but require further investigation to identify the optimum conditions for their use in testing of susceptibility profiles of filamentous fungi to antifungal agents.

\section{Introduction}

The impact and severity of fungal infections have grown in recent decades and now involve a wide range of fungal pathogens as etiological agents. During this period, the number of immunocompromised patients has markedly increased [1]. Many predisposing factors have contributed to this increase such as use of new and more aggressive therapies for treatment of solid tumours, haematological malignancies, long-term therapy with corticosteroids and broad-spectrum antibiotics, the increasing number of patients who undergo organ transplantation, and finally the spread of AIDS [2]. The management of fungal infections is affected by the ability to carry out rapid and effective etiological diagnosis and availability of antifungal agents with proper spectrum of activity [3]. Delayed diagnosis and antifungal treatment contribute significantly to the high mortality rates 
associated with invasive fungal infections [4], whereas early intervention with antifungal drugs may result in more effective management of high-risk patients [5].

Knowledge of potential causative organisms is required to aid the diagnostic process, mainly in situations where systemic fungal infection is suspected but the clinical presentation is nonspecific [6]. The exact identification of the etiological agent has become essential in light of the increased use of prophylactic regimens that predispose the patients' development of fungal infection and selection of resistant fungal species, such as Candida glabrata, C. krusei, Aspergillus terreus, Scedosporium species and Zygomycetes, many of which are intrinsically resistant to the available antifungal agents [7].

It is therefore very important to perform in vitro antifungal susceptibility testing (AFST) which should provide useful information for appropriate selection of the most active antifungal therapy against different etiological agents, as well as to predict treatment outcome or explain some resistance cases [8]. In 2002, the CLSI (Clinical and Laboratory Standards Institute, formerly NCCLS) published approved guidelines (M38-A) for broth microdilution susceptibility testing of Aspergillus spp. and other rapidly growing filamentous moulds (BMD) [9]. With the establishment of this reference method, there is now an opportunity to validate alternative methods for testing moulds against various systemically active antifungal agents. However this method is not a practical testing tool for a busy microbiology laboratory, because it is time-consuming and cumbersome [10].

Therefore, there is a great need for an easier and reproducible method for in vitro AFST of filamentous fungi. Alternatives to the CLSI method are E-test and Sensititre YeastOne (SYO) antifungal panel, two commercial methods that have been evaluated for yeasts and moulds [11-16]. SYO is a commercial colorimetric panel, that consists of a disposable tray which contains dried serial dilutions of seven antifungal agents in individual wells, which also contain an oxidation-reduction indicator (AlamarBlue) to generate clear-cut endpoints based on a visually detectable change in colour from blue to pink, and this indicates the antifungal activity, reducing the problems caused by the trailing effect in the visual endpoints $[11,14,16]$. The agar-based E-test method ( $A B$ Biomerieux, France) is a commercially available, pre-formed, pre-defined and stable antibiotic method, which uses a plastic test strip impregnated with a continuous concentration gradient of an antifungal agent to determine the minimum inhibitory concentration (MIC) necessary for inhibition of fungal growth [15-17].
It has been proven as a useful method in testing Candida spp. against a variety of antifungal agents, including amphotericin B, the azoles and caspofungin [8, 18-20].

The aim of this study was to evaluate the suitability of SYO method for susceptibility profiles' determination of non-Aspergillus moulds and of E-test for Aspergillus spp. and non-Aspergillus moulds, and, where available, other methods. The study was performed at the Laboratory for Medical Mycology at the Institute for Medical Microbiology, University of Zürich, Switzerland.

\section{Material and Methods}

\section{Test isolates}

In this study, a total of 33 clinical isolates belonging to different species of filamentous fungi from the collection of the mycology laboratory (Aspergillus fumigatus (9), A. glaucus (2), A. flavus (1), A. niger (1), A. nidulans (1), A. sclerotiorum (1) A. versicolor (1), Fusarium spp. (5), Paecilomyces variotii (1), Paecilomyces spp. (3), Scedosporium apiospermum (5), Scedosporium prolificans (2), Scopulariopsis spp. (1)), were tested during a fellowship stay at the Laboratory for Medical Mycology at the Institute for Medical Microbiology, University of Zürich, Switzerland in 2006.

Minimum inhibitory concentrations have been determined with Sensititre YeastOne (SYO) method (for Aspergillus spp.) and BMD method (for non-Aspergillus moulds). Culture and identification of the strains were done by using conventional mycological methods. All isolates were stored in skim milk at $-70^{\circ} \mathrm{C}$ until they were used in the study. They were restored and subsequently passed at least twice on potato carrot agar (nonAspergillus moulds) and malt yeast agar (Aspergillus spp.) to ensure viability and adequate sporulation before AFST with E-test (Aspergillus spp. and non-Aspergillus moulds) and SYO (non-Aspergillus moulds) was performed. Inoculum suspensions were prepared from 5-7 days cultures in sterile saline solution with Tween 80 for SYO method as well as for E-test. These suspensions were vortexed for 15 seconds to allow complete uniformity and left for another 15 minutes to allow sedimentation of hyphae. Final inocula were achieved by counting the number of conidia in a Neubauer chamber and were in the range between $0.5 \times 10^{6}-5 \times 10^{6} \mathrm{CFU} / \mathrm{ml}$ for SYO method and E-test. A confirmatory plate count was done from a 1:1000 dilution to ensure inoculum density on brain heart infusion agar (expected colony forming units $(C F U)=15-80$; all inocula were in the range between 15- 
80 CFU). The quality control (QC) strain C. parapsilosis ATCC 22019 (9) was included as control strain for both methods.

\section{M38-A reference broth microdilution method (BMD)}

A broth microdilution was performed according to the CLSI document M38-A using colorimetric endpoints. The antifungal drugs used in the study were obtained as reagent-grade powders: amphotericin B (AB) (Sigma Chemical Co.), itraconazole (IZ) (Janseen

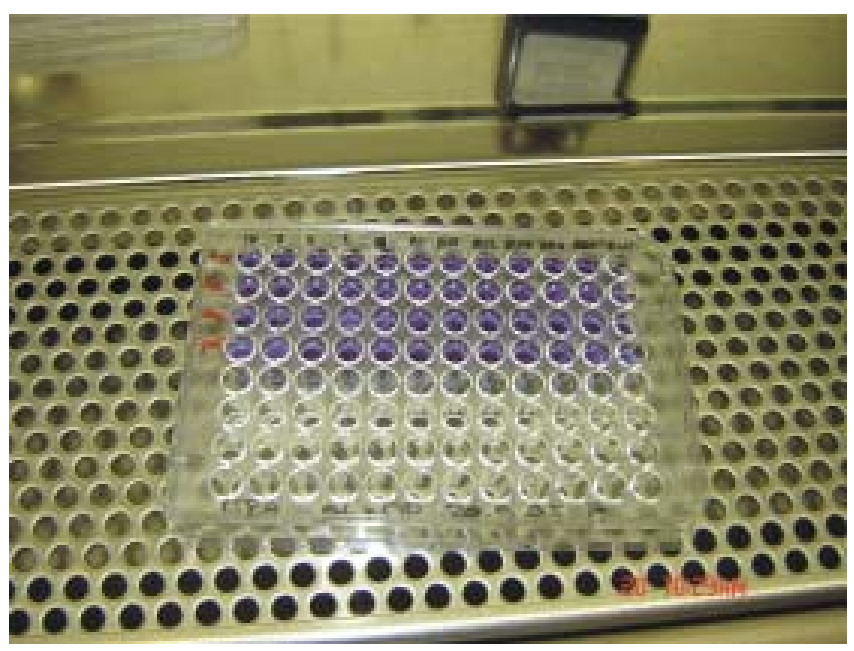

Figure 1: Microdilution trays for the reference broth microdilution method.

Pharmaceutica), voriconazole (VZ) (Pfizer). Stock solutions of the antifungal agents were prepared in 100\% dimethyl sulfoxide and diluted 100 times to their final concentration, further diluted in RPMI 1640 medium, for all antifungal agents (except for amphotericin B, for which AM3 was used), buffered to $\mathrm{pH} 7.0$ with MOPS buffer, and dispensed into 96-well microdilution trays (Fig. 1). The final concentration of the antifungal agents in the wells ranged from 0.06 to $16 \mu \mathrm{g} / \mathrm{ml}$ (amphotericin
$B$, itraconazole), from 0.03 to $8 \mu \mathrm{g} / \mathrm{ml}$ (voriconazole), and from 0.06 to $16 \mu \mathrm{g} / \mathrm{ml}$ (caspofungin). The inoculated microdilution trays containing Alamar blue were incubated at $35^{\circ} \mathrm{C}$ and read at 24,48 and 72 hours. Growth of filamentous fungi was evident as a change in the colorimetric growth indicator from blue (negative) to red (positive). The MIC endpoint was defined as the lowest concentration that produced complete inhibition of growth, i.e., the first blue well (for amphotericin B) or the lowest concentration that substantially inhibited growth, i.e., the first well which changed colour from blue to pink or red (for azoles and caspofungin) [9].

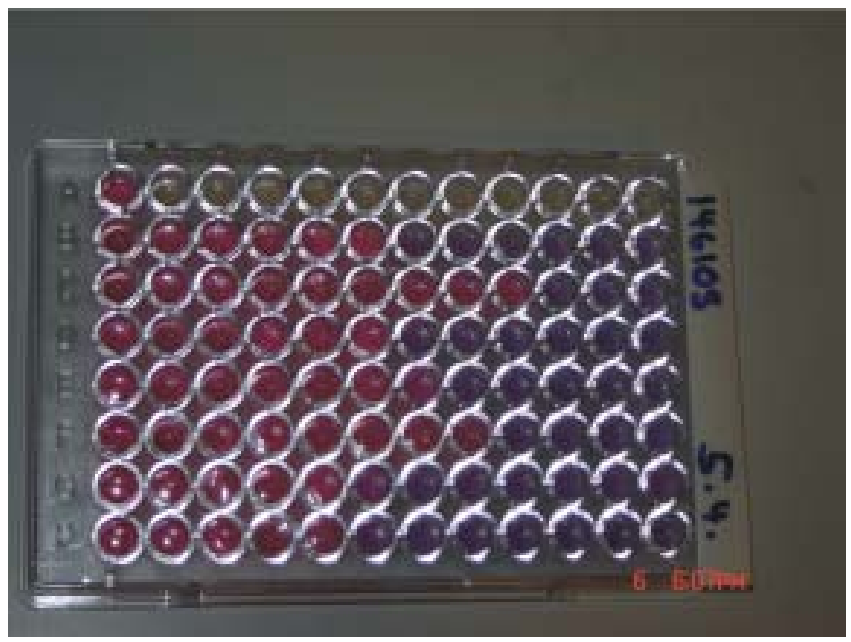

Figure 2: SYO5 Sensititre panels.

\section{Sensititre Yeast One method (SYO method)}

SYO5 panels (Trek Diagnostic System, UK) with incorporated Alamar blue containing twofold serial dilutions of dried antifungal drugs were used (Fig. 2). The concentration range for each agent is as follows (Amphotericin B $=0.008-16 \mu \mathrm{g} / \mathrm{ml}$; Fluconazole $=0.125$ $256 \mu \mathrm{g} / \mathrm{ml}$; Itraconazole $=0.008-16 \mu \mathrm{g} / \mathrm{ml}$; Ketoconazole $=0.008-16 \mu \mathrm{g} / \mathrm{ml} ;$ Flucytosine $=0.03-64 \mu \mathrm{g} / \mathrm{ml}$;
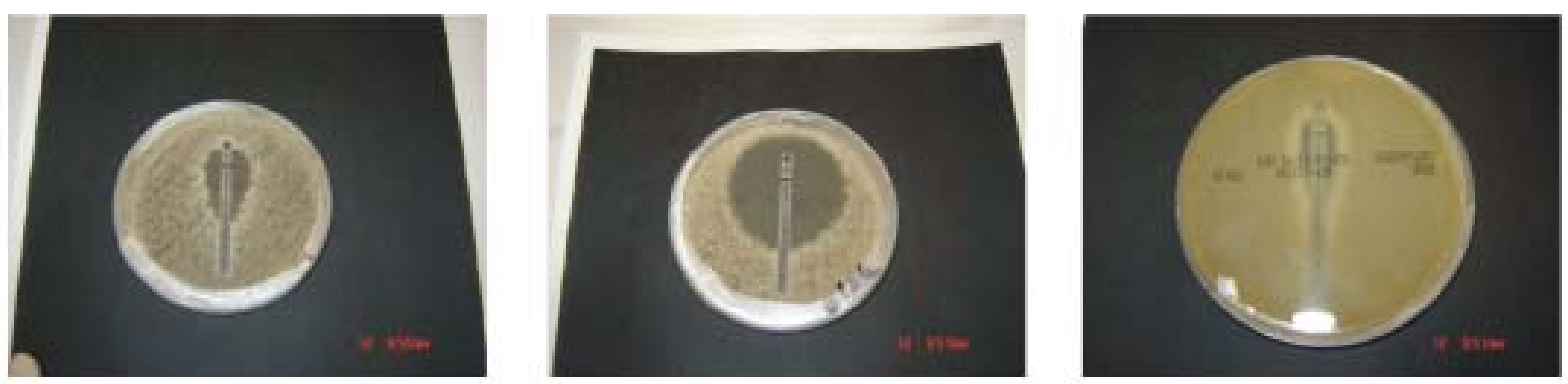

Figure 3: E-test gradient strips of different antifungal agents. 
Voriconazole $=0.008-16 \mu \mathrm{g} / \mathrm{ml} ;$ Caspofungin $=0.008$ $16 \mu \mathrm{g} / \mathrm{ml})$. Inoculum suspensions were diluted 1:100 in YeastOne RPMI medium, the dried SYO panels were rehydrated with $100 \mathrm{ml}$ of the working suspension which was dispensed into each well with a multichannel pippeting device. The panels were covered with seal strips and incubated at $35^{\circ} \mathrm{C}$ for 24,48 and 72 hours in a non- $\mathrm{CO}_{2}$ incubator and then visually read under normal laboratory lighting. Growth of filamentous fungi was evident as a change in the colorimetric growth indicator from blue (negative) to red (positive). The colorimetric MIC was considered to be the lowest concentration of an antifungal agent without any change in the colour (i.e., the first blue well) [11].

\section{E-test method}

The E-test method was performed in accordance with the manufacturer's instructions. E-test gradient strips of amphotericin B (AB), itraconazole (IZ), voriconazole (VZ), and caspofungin (CS) were obtained from Axon Lab AG, Baden, Switzerland, posaconazole (PZ) was obtained from Essex Chemie AG, Luzern, Switzerland. The strips were stored frozen on $-20^{\circ} \mathrm{C}$ until they were used in the study. Before AFST, they were left on room temperature for 30 minutes. The agar formulation used for the E-test was RPMI 1640, supplemented with $1.5 \%$ agar and $2 \%$ glucose and buffered to $\mathrm{pH} 7.0$ with $0.165 \mathrm{M}$ morpholine propanesulfonic acid (MOPS) buffer. The $90-\mathrm{mm}$ diameter plates contained RPMI at a depth of $4.0 \mathrm{~mm}$. The plates were inoculated by dipping a sterile swab into the cell suspension and streaking it across the surface of the agar in three directions. The plates were dried at ambient temperature for 15 minutes before applying the E-test strips. The plates with the E-test strips were incubated at $35^{\circ} \mathrm{C}$ and read at 24 or 48 hours for Aspergillus spp. (depending on the species growth) and at 48 or 72 hours for other non-Aspergillus moulds. The E-test MIC of azoles and caspofungin was considered as the drug concentration at the point where dense colonial growth intersected the strip, ignoring sparse subsurface hyphae at the margins. For amphotericin B, a complete inhibition of growth was read. Microcolonies within the ellipse were ignored [13].

\section{Data analysis}

E-test MICs read at $48 \mathrm{~h}$ were compared to BMD and SYO MICs read at $48 \mathrm{~h}$. Since the E-test scale has a continuous gradient of concentrations, the MICs with the E-test in between twofold dilutions were rounded to the next higher level for comparison [12-14]. For those strains in which the difference in MICs with the BMD was more than two-fold dilutions, MICs were repeated by SYO and E-test.

\section{Results}

The susceptibility profile of different Aspergillus spp. to antifungal agents was determined with E-test; results were compared with previous results of SYO

Table 1: In vitro susceptibility of Aspergillus spp. a tested by SYO, and E-test methods.

\begin{tabular}{|c|c|c|c|c|c|}
\hline \multirow{2}{*}{$\begin{array}{l}\text { Organism } \\
\text { (no. Isolates) }\end{array}$} & \multirow[t]{2}{*}{ Antifungal } & \multicolumn{2}{|l|}{ SYO } & \multicolumn{2}{|c|}{ E-test } \\
\hline & & Range & $\mathrm{MIC}_{50}{ }^{1)}$ & Range & $\mathrm{MIC}_{50}{ }^{1)}$ \\
\hline \multirow{8}{*}{$\begin{array}{l}\text { A. fumigatus } \\
\text { (9) }\end{array}$} & Amphotericin B & $0.5-2.0$ & 1.0 & $0.19-4.0$ & 2.0 \\
\hline & Fluconazole & $128-7256$ & 7256 & - & - \\
\hline & Itraconazole & $0.032-0.5$ & 0.125 & $0.19-0.5$ & 0.38 \\
\hline & Ketoconazole & $0.5-8.0$ & 2.0 & - & - \\
\hline & Posaconazole & - & . & $0.094-0.125$ & 0.094 \\
\hline & Voriconazole & $0.064-0.5$ & 0.25 & $0.064-0.125$ & 0.125 \\
\hline & 5-Fluorocytosine & $0.5-764$ & 64.0 & - & - \\
\hline & Caspofungin & " & & $0.047-0.125$ & 0.094 \\
\hline \multirow[t]{2}{*}{ A. glaucus } & Amphotericin B & $1.0-1.0$ & & $0.094-2.0$ & \\
\hline & Fluconazole & $64-7256$ & - & - & - \\
\hline \multirow[t]{6}{*}{ (2) } & Itraconazole & $0.008-$ & - & $0.004-$ & - \\
\hline & Ketoconazole & $0.016-4.0$ & - & 0.120 & - \\
\hline & Posaconazole & - & - & $0.004-0.064$ & - \\
\hline & Voriconazole & $0.064-0.5$ & - & $0.002-0.023$ & - \\
\hline & 5-Fluorocytosine & $8.0-32.0$ & - & - & - \\
\hline & Caspofungin & " & & $0.044-0.125$ & \\
\hline \multirow{8}{*}{$\begin{array}{l}\text { A. flavus } \\
\text { (1) }\end{array}$} & Amphotericin B & 2.0 & & 6.0 & \\
\hline & Fluconazole & $>256$ & - & - & - \\
\hline & Itraconazole & 0.125 & - & 0.25 & - \\
\hline & Ketoconazole & 1.0 & - & - & - \\
\hline & Posaconazole & - & - & 0.25 & - \\
\hline & Voriconazole & 0.125 & - & 0.25 & - \\
\hline & 5-Fluorocytosine & 2.0 & - & - & - \\
\hline & Caspofungin & $"$ & & 0.16 & \\
\hline \multirow{8}{*}{$\begin{array}{l}\text { A. sclerotiorum } \\
\text { (1) }\end{array}$} & Amphotericin B & 4.0 & & 4.0 & \\
\hline & Fluconazole & $>256$ & - & & - \\
\hline & Itraconazole & 0.5 & - & 0.5 & - \\
\hline & Ketoconazole & 4.0 & - & - & - \\
\hline & Posaconazole & - & - & 0.125 & - \\
\hline & Voriconazole & 0.25 & - & 0.125 & - \\
\hline & 5-Fluorocytosine & $>64$ & - & - & - \\
\hline & Caspofungin & & & 0.094 & \\
\hline \multirow{8}{*}{$\begin{array}{l}\text { A. niger } \\
\text { (1) }\end{array}$} & Amphotericin B & 1.0 & & 0.5 & \\
\hline & Fluconazole & $>256$ & - & - & - \\
\hline & Itraconazole & 0.5 & - & 2.0 & - \\
\hline & Ketoconazole & 4.0 & - & - & - \\
\hline & Posaconazole & & - & 0.25 & - \\
\hline & Voriconazole & 0.5 & - & 0.38 & - \\
\hline & 5-Fluorocytosine & 64.0 & - & - & - \\
\hline & Caspofungin & & & 0.032 & \\
\hline \multirow{8}{*}{$\begin{array}{l}\text { A. nidulans } \\
\text { (1) }\end{array}$} & Amphotericin B & 2.0 & - & 1.0 & - \\
\hline & Fluconazole & 128 & - & - & - \\
\hline & Itraconazole & 0.125 & - & 0.125 & - \\
\hline & Ketoconazole & 0.125 & - & - & - \\
\hline & Posaconazole & & & 0.125 & \\
\hline & Voriconazole & 0.064 & - & 0.125 & - \\
\hline & 5-Fluorocytosine & $>64$ & - & - & - \\
\hline & Caspofungin & & & $>16$ & \\
\hline \multirow{8}{*}{$\begin{array}{l}\text { A. versicolor } \\
\text { (1) }\end{array}$} & Amphotericin B & 4.0 & & 4.0 & \\
\hline & Fluconazole & $>256$ & - & - & - \\
\hline & Itraconazole & 0.25 & - & 0.25 & - \\
\hline & Ketoconazole & 0.5 & - & - & - \\
\hline & Posaconazole & - & - & 0.094 & - \\
\hline & Voriconazole & 0.125 & - & 0.125 & - \\
\hline & 5-Fluorocytosine & $>64$ & - & - & - \\
\hline & Caspofungin & - & - & 0.094 & - \\
\hline
\end{tabular}

-, Not determined; 1 ) $\mathrm{MIC}_{50}$ (MIC causing inhibition of $50 \%$ of isolates) values were calculated for those species with 3 or more isolates. 
method. For non-Aspergillus moulds SYO and E-test were performed, and results were compared with previous results of CLSI BMD method. All strains produced detectable growth after $48 \mathrm{~h}$ of incubation, except one strain of $A$. glaucus and one strain of Paecilomyces spp. that required 6 and 7 days of incubation respectively for growth in SYO plates. The susceptibility testing was performed twice for these strains to ensure avoidance of technical errors. Results with control strain were within the described range according to reference documents [9]. E-test inhibition ellipses were clear. Triazole ellipses, especially those of voriconazole were wider than amphotericin B ellipses for most isolates. Tables 1 and 2 summarize the antifungal susceptibility profiles of the 33 strains of Aspergillus spp. and non-Aspergillus moulds to amphotericin B, itraconazole, voriconazole, posaconazole and caspofungin obtained with the E-test, to amphotericin $B$, fluconazole, itraconazole, ketoconazole, flucytosine and voriconazole as determined by the SYO method and to amphotericin $\mathrm{B}$, itraconazole, voriconazole and caspofungin as determined with the CLSI BMD.

\section{SYO versus E-test}

In 9/33 (27\%) mould isolates amphotericin B MICs were found to be identical by SYO and E-test, in 12/ $33(36 \%)$ isolates amphotericin B MICs differed by \pm 1 dilution, and in 7 isolates $(21 \%)$ they differed by \pm 2 dilutions (Table 3). Differences were most frequently due to higher MICs with the E-test. Discrepancies (greater than \pm 2 dilutions) between the MICs determined by the SYO method and E-test were demonstrated in 5/33 (15\%) isolates tested against amphotericin B: 1 A. glaucus, 1 Scedosporium prolificans, 2 Scedosporium apiospermum, and 1 Paecilomyces spp., and these discrepancies were almost always due to higher MICs with the E-test (except for A. glaucus, for which E-test result was lower). Overall, 4 strains were classified as amphotericin B "resistant" (MIC>32 $\mu \mathrm{g} / \mathrm{ml}$ ) by the Etest, but SYO method failed to detect these strains. In 16 out of $33(48 \%)$ moulds itraconazole MICs were determined as identical by both methods, in $11 / 33(33 \%)$ isolates the itraconazole MICs differed by \pm 1 dilution with E-test, and in $4 / 33(12 \%)$ the itraconazole MICs were two dilutions higher by the E-test. Two (6\%) major discrepancies between the itraconazole MICs with both methods were observed. For voriconazole, in 11/33 (33\%) voriconazole MICs were found to be identical by both methods, in 13/ $33(39 \%)$ isolates the voriconazole MICs differed by \pm 1 dilution. In 4/33 (12\%) the voriconazole MICs differed by two dilutions. In 5 (15\%) strains major discrepancies with
Table 2: In vitro susceptibility of non-Aspergillus moulds a tested by SYO, E-test, and BMD methods.

\begin{tabular}{|c|c|c|c|c|c|c|c|}
\hline \multirow{2}{*}{$\begin{array}{l}\text { Organism (no. } \\
\text { Isolates) }\end{array}$} & \multirow{2}{*}{ Antifungal } & \multicolumn{2}{|c|}{ SYO } & \multicolumn{2}{|c|}{ E-test } & \multicolumn{2}{|c|}{ BMD } \\
\hline & & Range & $\mathrm{MIC}_{50}{ }^{1)}$ & Range & $\mathrm{MIC}_{50}{ }^{1)}$ & Range & $\mathrm{MIC}_{50}{ }^{1)}$ \\
\hline \multirow{8}{*}{ Fusarium sp. (5) } & Amphotericin B & $0.25-2.0$ & 1.0 & $0.25-4.0$ & 1.5 & $2.0-8.0$ & 4.0 \\
\hline & Fluconazole & $\geq 256-\geq 256$ & $\geq 256$ & - & - & - & - \\
\hline & Itracon azole & $\geq 16-\geq 16$ & $\geq 16$ & $\geq 32-\geq 32$ & $\geq 32$ & $16-\geq 16$ & $\geq 16$ \\
\hline & Ketoconazole & $8.0-\geq 16$ & $\geq 16$ & - & - & - & - \\
\hline & Posaconazde & - & - & $8.0-\geq 32$ & $\geq 32$ & - & - \\
\hline & Voricon azole & $1.0-4.0$ & 4.0 & $2.0-4.0$ & 4.0 & $4.0-\geq 8.0$ & 4.0 \\
\hline & 5-Fluorocytcsine & $\geq 64-\geq 64$ & $\geq 64$ & - & - & - & - \\
\hline & Caspofungin & - & - & $\geq 32-\geq 32$ & $\geq 32$ & $0.12-\geq 16$ & 4.0 \\
\hline \multirow{7}{*}{$\begin{array}{l}\text { Scedosporium } \\
\text { apiospermum (5) }\end{array}$} & Amphotericin B & $2.0-\geq 16$ & 2.0 & $4.0-\geq 32$ & 32 & $4.0-\geq 16$ & $\geq 16$ \\
\hline & Fluconazole & $16-32$ & 16 & & & & \\
\hline & Itracon azole & $0.064-0.5$ & 0.5 & $0.25-2.0$ & 0.5 & $0.5-2.0$ & 1.0 \\
\hline & Ketoconazole & $0.125-0.5$ & 0.25 & - & & - & - \\
\hline & Posaconazde & & - & $0.25-2.0$ & 0.5 & - & \\
\hline & Voricon azole & $\begin{array}{c}0.064-0.125 \\
>64->64\end{array}$ & $\begin{array}{c}0.125 \\
>64\end{array}$ & $0.064-0.64$ & 0.125 & $0.12-0.25$ & 0.25 \\
\hline & Caspofungin & - & - & $\geq 32-\geq 32$ & $\geq 32$ & - & - \\
\hline \multirow{8}{*}{$\begin{array}{l}\text { Scedosporium } \\
\text { prolificans (2) }\end{array}$} & Amphotericin B & $4.0-\geq 16$ & - & $\geq 32-\geq 32$ & - & $16-\geq 16$ & - \\
\hline & Fluconazole & $\geq 256-\geq 256$ & - & & - & - & \\
\hline & Itraconazole & $\geq 16-\geq 16$ & - & $\geq 32-\geq 32$ & - & - & - \\
\hline & Ketoconazole & $4.0-\geq 16$ & - & - & - & - & - \\
\hline & Posaconazole & - & - & $0.25-\geq 32$ & - & - & - \\
\hline & Voricon azole & $2.0-16$ & - & $1.5-\geq 24$ & - & $1.0-2.0$ & - \\
\hline & 5-Fluoro cytosi ne & $\geq 64-\geq 64$ & - & - & - & - & - \\
\hline & Caspofungin & - & - & $\geq 32-\geq 32$ & - & - & - \\
\hline \multirow{8}{*}{$\begin{array}{l}\text { Paecilomyces sp. } \\
\text { (3) }\end{array}$} & Amphotericin B & $2.0-\geq 16$ & 2.0 & $2.0-\geq 32$ & $\geq 32$ & $8.0-\geq 16$ & $\geq 16$ \\
\hline & Fluconazole & $32-\geq 256$ & $\geq 256$ & - & - & - & - \\
\hline & Itracon azole & $1.0-\geq 16$ & $\geq 16^{2)}$ & $0.75-\geq 32$ & $\geq 32$ & $8.0-\geq 16$ & $\geq 16$ \\
\hline & Ketoconazole & $2.0-\geq 16$ & - & - & - & - & - \\
\hline & Posaconazde & - & -2 & $1.0-2.0$ & 2.0 & - & - \\
\hline & Voricon azole & $0.25-\geq 16$ & $1.0^{2}$ & $0.125-\geq 32$ & 0.64 & $0.5-1.0$ & 1.0 \\
\hline & 5-Fluoro cytosine & $0.03-2.0$ & - & - & - & - & - \\
\hline & Caspofungin & (1) & - & $0.16-\geq 32$ & $\geq 32$ & - & - \\
\hline \multirow{8}{*}{$\begin{array}{l}\text { Paecilomyces } \\
\text { varioti (1) }\end{array}$} & Amphotericin B & 0.5 & - & 0.125 & - & 0.5 & - \\
\hline & Fluconazole & $\geq 256$ & - & & & & \\
\hline & Itracon azole & 0.064 & - & 0.094 & - & 0.5 & - \\
\hline & Ketoconazole & 1.0 & - & & - & - & - \\
\hline & Posaconazde & - & - & 0.094 & - & - & - \\
\hline & Voriconazole & 8.0 & - & 8.0 & - & 8.0 & - \\
\hline & 5-Fluoro cytcsine & 0.25 & - & & - & - & - \\
\hline & Caspofungin & & - & 0.012 & - & - & - \\
\hline \multirow{8}{*}{$\begin{array}{l}\text { Scopulariopsis } s p \text {. } \\
\text { (1) }\end{array}$} & Amphotericin B & 4.0 & - & $\geq 16$ & - & $\geq 16$ & - \\
\hline & Fluconazole & $\geq 256$ & - & - & - & - & - \\
\hline & Itracon azole & $\geq 16$ & - & $\geq 32$ & - & $\geq 16$ & - \\
\hline & Ketoconazole & 0.5 & - & - & - & - & - \\
\hline & Posaconazde & - & - & $\geq 32$ & - & - & - \\
\hline & Voriconazole & 4.0 & - & $\geq 32$ & - & 8.0 & - \\
\hline & 5-Fluorocytcsine & $\geq 64$ & - & - & - & - & - \\
\hline & Caspofungin & & - & 24 & - & - & - \\
\hline
\end{tabular}

-, Not determined; 1 ) $\mathrm{MIC}_{50}$ (MIC causing inhibition of $50 \%$ of isolates) values were calculated for those species with 3 or more isolates; 2) MIC $C_{50}$ was not determined because 1 of 3 values was not determined.

more than 2 dilution higher values in favour of E-test were registered.

\section{SYO versus $M-B M D$}

In $4 / 17$ (24\%) non-Aspergillus moulds, amphotericin B MICs were determined to be identical by SYO method and BMD, in 3/17 (18\%) isolates amphotericin B MICs differed by one dilution, and in 6/17 (35\%) MICs for amphotericin B differed by two dilutions higher. Where differences by one or two dilutions were observed, they were due to higher MICs with BMD. In 4 (24\%) strains, major discrepancies with more than 2 dilutions higher values occurred and they were due to higher MICs with BMD. In 4/17 (24\%) isolates of nonAspergillus moulds, the itraconazole MICs were identical by both methods, in $3 / 17(18 \%)$ isolates differed by \pm 1 dilution, and in 1 (6\%) isolate by two dilutions. In 4 (24\%) 
strains, major discrepancies with more than 2 dilutions were noticed. In 5/17 (29\%) the MIC with BMD was not determined (data not shown). In 4/17 (24\%) isolates of non-Aspergillus moulds, the voriconazole MICs were determined identical by both methods, in 10/17 (59\%) isolates they differed by \pm 1 dilution, and in one (6\%) isolate by two dilutions. In $2(12 \%)$ strains, major discrepancies with more than 2 dilutions were noticed.

Table 3: Agreement between SYO and E-test, BMD and SYO and $M-B M D$ and E-test for three agents tested against 33 isolates of Aspergillus spp. and non- Aspergillus moulds.

\begin{tabular}{llcc}
\hline \multirow{2}{*}{$\begin{array}{l}\text { Agreement between } \\
\text { methods }\end{array}$} & $\begin{array}{l}\text { Antifungal } \\
\text { agent }\end{array}$ & \multicolumn{2}{c}{$\%$ Agre ement } \\
\cline { 3 - 4 } & & $\begin{array}{c} \pm 1 \\
\text { dilution }\end{array}$ & $\begin{array}{c} \pm 2 \\
\text { dilutions }\end{array}$ \\
\hline \multirow{2}{*}{ SYO and E-test $(\mathrm{n}=33)$} & $\begin{array}{l}\text { Amphotericin B } \\
\text { Itraconazole }\end{array}$ & 63.6 & 84.9 \\
& Voriconazole & 72.7 & 93.9 \\
& & 84.8 \\
BMD and SYO $(\mathrm{n}=17)$ & Amphotericin B & 41.2 & 76.5 \\
& Itraconazole & 58.3 & 66.7 \\
& Voriconazole & 82.4 & 88.2 \\
& & & \\
& Amphotericin B & 47.1 & 82.4 \\
BMD and E-test $(\mathrm{n}=17)$ & Itraconazole & 58.3 & 83.3 \\
& Voriconazole & 64.7 & 82.4 \\
\hline
\end{tabular}

\section{E-test versus $M B D$}

In 7/17 (41\%) isolates of non-Aspergillus moulds, amphotericin B MICs were determined to be same by both methods, in $1(6 \%)$ isolate amphotericin B MIC differed by \pm 1 , and in $6 / 17$ (35\%) amphotericin B MIC differed by \pm 2 dilutions. Discrepancies (greater than \pm 2 dilutions) between the MICs determined by BMD and Etest were demonstrated in 3/17 (18\% tested against amphotericin B. In 6/17 (35\%) isolates of non-Aspergillus moulds, itraconazole MICs were determined to be identical by both methods, in $1(6 \%)$ isolate itraconazole MIC differed by \pm 1 dilution, and in $3 / 17$ (18\%) isolates they differed by \pm 2 dilutions. Discrepancies (greater than \pm 2 dilutions) between the MICs determined by the BMD and E-test were demonstrated in 2/17 (12\%) tested against itraconazole. In 5/17 (29\%) the MIC with the BMD was not determined (data not shown). In 4/17 $(24 \%)$ isolates of non-Aspergillus moulds, voriconazole MICs were determined to be identical by both methods, in $7 / 17(41 \%)$ isolates voriconazole MICs differed by \pm 1 dilution, and in $3(18 \%)$ isolates voriconazole MIC differed by \pm 2 dilutions. Major discrepancies (greater than \pm 2 dilutions) between the MICs determined by BMD and Etest were demonstrated in 3/17 (18\%) tested against voriconazole (always due to higher MICs with E-test).
The MIC results obtained by both BMD and Etest methods demonstrated that voriconazole was very active against this diverse array (5 different genera) of opportunistic non-Aspergillus moulds (69.7\% susceptible at MIC $<1 \mu \mathrm{g} / \mathrm{ml}$ ). Elevated MICs as determined by all methods were more frequently observed with Fusarium spp., as well as with isolates of Scopulariopsis spp. and Paecilomyces spp. When discrepancies between the BMD and E-test MICs were noted, the E-test tended to give lower values with voriconazole. All isolates of Aspergillus spp. were inhibited with $<0.5 \mu \mathrm{g} / \mathrm{ml}$ of voriconazole. These results demonstrate the excellent efficacy of voriconazole against Aspergillus species and suggest that voriconazole may be the treatment of choice in invasive aspergillosis caused by A.fumigatus and A. flavus. Indeed, this is supported by clinical studies, which have shown better responses to voriconazole than to amphotericin B in patients with invasive aspergillosis (21-24).

\section{Discussion}

We found the E-test to be a very simple method for determination of susceptibility profile of filamentous moulds to all antifungals, except of few difficulties experienced with caspofungin, where residual growth was noticed. RPMI agar with glucose (final concentration, $2 \%$ ) supported optimal growth of all species tested and provided good agreement with the MICs obtained with BMD. Szekely et al. [25] used RPMI agar and $48 \mathrm{~h}$ of incubation and found that the inhibition ellipses were clear for most isolates. They concluded that the E-test procedure was reproducible and served as a suitable method for AFST of moulds. Pfaller et al. demonstrated that the E-test was able to detect resistance to itraconazole among filamentous fungi [15]. Additionally, the depth of the agar can influence the MIC. Therefore, the manufacturer's recommendations should be followed when attempting to obtain MICs by E-test. The SYO method has the advantage of being technically easy to perform and able to determine objective and quantitative MIC endpoints [27]. The BMD method is regarded as the reference method, however, a main disadvantage of this procedure is that the preparation of the trays is time consuming and laborious.

The results of this study show that the overall agreement at \pm 2 dilutions between the three methods is relatively high. However, the correlation of the SYO method to the BMD method is only moderate for amphotericin $\mathrm{B}$ and itraconazole. The vast majority of 
differing results are due to higher MICs with the BMD method. In particular, SYO was unable to detect some of the potentially amphotericin B resistant strains. Likewise, when comparing the results of the SYO method to the Etest method for amphotericin B and itraconazole, the majority of differing results are in favour of higher MICs with the E-test. Similarly, Meletiadis et al. [14] have shown lower SYO MICs for itraconazole in several Aspergillus spp. (except $A$. ustus). They could not show this effect for amphotericin B. In contrast, in another study in which also E-test, SYO and BMD were compared [27], MIC ranges by E-test were broader and always higher for amphotericin B and itraconazole.

The MIC results obtained by E-test methods demonstrated that posaconazole was very active against all Aspergillus spp. (all susceptible at MIC $<0.25 \mu \mathrm{g} / \mathrm{ml}$ ), but showed poor activity against some of the nonspergillus moulds (Fusarium spp., Scedosporium spp., Scopulariopsis spp.) $(28,29)$. SYO method includes fluconazole and 5-flucytosine, to which in general filamentous fungi are resistant and are not useful for therapy (panel testing thus generates more costs). Also, with SYO it is not possible to test caspofungin for moulds, because a majority of the strains appear resistant, whereas E-test allows testing of antifungal drugs according to the needs of the clinician (including caspofungin and posaconazole). However, SYO has potential value for the performance of susceptibility testing of filamentous fungi to other antifungal agents, such as itraconazole and voriconazole, so it could be a good and reliable alternative for in vitro AFST, especially with SYO8 which includes the newer triazole, posaconazole. But the results provided with this study indicate that SYO is not sensitive enough for in vitro detection of resistance to amphotericin B in Aspergillus spp. and non-Aspergillus moulds.

In conclusion, on the basis of data from this study and other studies as well, the ability of E-test to generate MIC data for filamentous fungi that are comparable to those obtained by the CLSI broth microdilution method has been shown and thus a potential value for E-test for the use for AFST of mould pathogens has been proved. This could be attractive to microbiology laboratories, since it will provide the flexibility to test one or more commonly used antifungal agents selectively against a wide variety of moulds that may be encountered clinically. The higher amphotericin B MICs obtained by the E-test as compared to SYO for some strains suggests that this method could be more useful in detection of mould isolates potentially resistant to amphotericin B. In general, both the E-test and the SYO methods are promising, but require further investigation to identify the optimum conditions for their use in the testing of the susceptibilities of filamentous fungi to antifungal agents, including new azoles and the new class of echinocandins.

Optimization of these tests might require adjustments depending on the species tested. However, more studies are required to determine which methods show the best agreement with in vivo results, and thus, the clinical value of these in vitro results should be determined in clinical trials.

\section{Acknowledgments}

We would like to express our sincere gratitude to the entire staff of the Laboratory for Medical Mycology, Institute for Medical Microbiology, Zurich, Switzerland for the tchnical support.

\section{References}

1. Zicker M, Colombo AL, Ferraz-Neto BH, Camargo LFA. Epidemiology of fungal infections in liver transplant recipients: a six-year study of a large Brazilian liver transplantation centre. Mem Inst Oswaldo Cruz. 2011;106(3):339-45.

2. Uppin MS, Anuradha SV, Uppin SG, Paul TR, Prayaga AK, Sundaram C. Fungal infections as a contributing cause of death: an autopsy study. Indian J Pathol Microbiol. 2011;54(2):344-9.

3. Posteraro B, Torelli R, Carolis E, Posteraro P, Sanguinetti $M$. Update on the Laboratory Diagnosis of Invasive Fungal Infections. Mediterr J Hematol Infect Dis. 2011;3(1):e2011002. Epub 2011 Jan 14.

4. Morrell M, Fraser VJ, Kollef MH. Delaying the empiric treatment of Candida bloodstream infection until positive blood culture results are obtained: a potential risk factor for hospital mortality. Antimicrob Agents Chemother. 2005;49:3640-3645.

5. Murali S, Langston A. Advances in antifungal prophylaxis and empiric therapy in patients with hematologic malignancies. Transpl Infect Dis. 2009;11:480-490.

6. Dismukes WE, Pappas PG, Sobel JD. Clinical Mycology. New York: Oxford University Press, 2003.

7. Rodriguez-Tudela JL, Alcazar-Fuoli L, Cuesta I, AlastrueyIzquierdo A, Monzon A, Mellado E, Cuenca-Estrella M. Clinical relevance of resistance to antifungals. Int J Antimicrob Agents. 2008;32:S111-113.

8. Perkhofer S, Mrazek C, Hartl L, Lass-Flörl C. In Vitro Susceptibility Testing in Fungi: What is its Role in Clinical 
Practice? Curr Infect Dis Rep. 2010;12(6):401-8.

9. National Committee for Clinical Laboratory Standards. Reference method for broth dilution antifungal susceptibility testing of conidium-forming filamentous fungi: approved standard M38-A. NCCLS, Wayne, PA, USA, 2002.

10. Lass-Flörl C. In vitro susceptibility testing in Aspergillus species: un update. Future Microbiol. 2010;5(5):789-99.

11. Carrilo-Munoz AJ, Quindos G, Del Valle O, HernandezMolina JM, Santos P. Antifungal activity of Amphotericin B and Itraconazole against filamentous fungi: comparison of the Sensititre YeastOne and NCCLS M38-A reference methods. J Chemotherapy. 2004;16:468-473.

12. Serrano MC, Morilla D et al. Comparison of E-test with modified broth microdilution method for testing susceptibility of Aspergillus spp. to Voriconazole. J Clin Microbiol. 2003;41:5270-5272.

13. Espinel-Ingroff A, Rezusta A. E-test method for testing susceptibilities of Aspergillus spp. to the new triazoles voriconazole and posaconazole and to established antifungal agents: comparison with NCCLS broth microdilution method. J Clin Microbiol. 2002; 40:2101-2107.

14. Meletiadis J, Mouton JS, Meis J, Bouman BA, Verweij PE and the EUROFUNG Network. Comparison of the Etest and the Sensititre colorimetric methods with the NCCLS proposed standard for antifungal susceptibility testing of Aspergillus species. J Clin Microbiol. 2002;40:2876-2885.

15. Pfaller MA, Messer SA, Mills K, Bolmström A. In vitro susceptibility testing of filamentous fungi: comparison of Etest and reference microdilution methods for determining itraconazole MICs. J Clin Microbiol. 2000;38:3359-3361.

16. Castro C, Carmen Serrano M et al. Comparison of the Sensititre Yeast One colorimetric antifungal panel with a modified NCCLS M38-A method to determine the activity of voriconazole against clinical isolates of Aspergillus spp. J Clin Microbiol. 2004;42:4358-4360.

17. Espinel-Ingroff A, Warnock DW, Vasquez JA, ArthimgtonSkaggs BA. In vitro antifungal susceptibility methods and clinical implications of antifungal resistance. Med Mycol. 2000;38:293-304.

18. Negri M, Henriques M, Svidzinski TI, Paula CR, Oliveira R. Correlation between Etest, disk diffusion, and microdilution methods for antifungal susceptibility testing of Candida species from infection and colonization. J Clin Lab Anal. 2009;23(5):324-30.
19. Arredondo-Garcí a JL, Amábile-Cuevas CF; RedMic2 Study Group. Susceptibility of Mexican isolates of yeasts and moulds to amphotericin B and triazole antifungals. J Infect Dev Ctries. 2009;3(5):398-401.

20. Fleck R, Dietz A, Hof H. In vitro susceptibility of Candida species to five antifungal agents in a German university hospital assessed by the reference broth microdilution method and Etest. Antimicrob Chemother. 2007;59(4):767-771.

21. Walsh TJ, Lutsar I, Driscoll T. et al. Voriconazole in the treatment of aspergillosis, scedosporiosis and other invasive fungal infections in children. Pediatr Infect Dis J. 2002;21:240248.

22. Denning DW, Ribaud R, Milpied N et al. Efficacy and safety of voriconazole in the the treatment of acute invasive aspergillosis. Clin Infect Dis. 2002;34:563-571.

23. Herbrecht R, Denning DW, Patterson T, et al. Voriconazole versus amphotericin $B$ for primary therapy of invasive aspergillosis. N Engl J Med. 2002;347:408-415.

24. Scott LJ, Simpson D. Voriconazole: a review of its use in the management of invasive fungal infections. Drugs. 2007;67(2):269-98.

25. Szekely A, Johnson EM, Warnock DW. Comparison of Etest and broth microdilution methods for antifungal drug susceptibility testing of molds. J Clin Microbiol. 1999;37:14801483.

26. Carrillo-Muñoz AJ, Quindós G, Ruesga M, del Valle $O$, Pemán J, Cantón E, Hernández-Molina JM, Santos P. In vitro antifungal susceptibility testing of filamentous fungi with Sensititre Yeast One. Mycoses. 2006;49(4):293-7.

27. Martí n-Mazuelos E, Pemán J, Valverde A, Chaves M, Serrano MC, Cantón E. Comparison of the Sensititre YeastOne colorimetric antifungal panel and Etest with the NCCLS M38A method to determine the activity of amphotericin B and itraconazole against clinical isolates of Aspergillus spp. J Antimicrob Chemother. 2003;52(3):365-70.

28. Patel R, Mendrick C, Knapp CC, Grist R, McNicholas PM. Clinical evaluation of the Sensititre YeastOne plate for testing susceptibility of filamentous fungi to posaconazole. J Clin Microbiol. 2007;45(6):2000-1.

29. Lass-Flörl C, Mayr A, Perkhofer S, Hinterberger G, Hausdorfer J, Speth C, Fille M. Activities of antifungal agents against yeasts and filamentous fungi: assessment according to the methodology of the European Committee on Antimicrobial Susceptibility Testing. Antimicrob Agents Chemother. 2008;52(10):3637-41. 